\title{
TCF7L2 and therapeutic response to sulfonylureas in patients with type 2 diabetes
}

Andreas Holstein ${ }^{1}$, Michael Hahn', Antje Körner ${ }^{2}$, Michael Stumvoll³, Peter Kovacs ${ }^{4^{*}}$

\begin{abstract}
Background: Variants in the TCF7L2 have been shown to be associated with an increased risk for type 2 diabetes (T2D). Since the association with diabetes could be explained by effects on insulin secretion, we investigated whether patients with diabetes risk alleles at rs7903146 might have an altered hypoglycaemic response to sulfonylureas (SUs).
\end{abstract}

Methods: We recruited 189 patients with T2D being treated with SUs and determined the rs 7903146 diabetes risk genotype. We used a logistic regression with secondary SU failure defined as an A1C $\geq 7.0 \%$ after 6 months of SU treatment.

Results: In univariate regression analyses, TCF7L2 genotype was the only predictor of SU treatment failure. The rs7903146 T allele was significantly more frequent in the group of patients who failed to respond to SU (36\%) than in the control group (26\%) $[P=0.046$; odds ratio (OR): $1.57(1.01-2.45)$ in an additive mode of inheritance].

Conclusions: Our data suggest that patients with diabetes risk alleles in TCF7L2 have an altered hypoglycaemic response to SUs resulting in earlier secondary failure.

\section{Background}

The TCF7L2-gene (TCF7L2; Transcription factor 7-like 2) encodes a transcription factor (Tcf-4) that is involved in the regulation of cellular proliferation and differentiation [1]. Variants in the TCF7L2 have initially been shown to be associated with an increased risk for type 2 diabetes (T2D) in a genome-wide analysis of the isolate population of Iceland [2]. The strongest associations with T2D with a clear gene dose effect were reported for the rs7903146 variant [3]. The initial findings have been replicated in independent studies in multiple ethnic populations and were summarized in a large global meta-analysis [4]. The risk alleles actually predicted the progression from impaired glucose tolerance to diabetes prospectively [5] and an increased severity of the disease [6] in adults. Also, TCF7L2 variants conferred a higher risk for early impairment of glucose metabolism emerging as soon as in childhood and adolescence [7]. Some clinical data suggested that the polymorphisms affected the capacity of pancreatic $\beta$-cells to secrete insulin

\footnotetext{
* Correspondence: peter.kovacs@medizin.uni-leipzig.de

${ }^{4}$ Interdisciplinary Centre for Clinical Research, University of Leipzig, Leipzig, Germany

Full list of author information is available at the end of the article
}

rather than aggravating insulin resistance [5,8-13], possibly by impaired $\beta$-cell proinsulin-processing [14]. This was further supported by expression data suggesting a putative role of TCF7L2 in $\beta$-cell differentiation [12]. Considering the role of TCF7L2 risk variants in insulin secretion, Pearson et al. [15] hypothesized that patients with diabetes risk alleles at rs12255372 and rs7903146 have an altered hypoglycaemic response to sulfonylureas (SUs) due to decreased $\beta$-cell function. They could indeed show that carriers of the diabetes risk alleles from the GoDART (Genetics of Diabetes Audit and Research Tayside) study were less likely to respond to SUs [15]. This study suggested that genetic variation in TCF7L2 can alter response to therapy in T2D. Since a causal phenotype-genotype relationship can not be established with one initial report, replication studies are the backbone to the genetic epidemiology of complex diseases [16], and play a crucial role in pharmacogenomics as well.

Therefore, we sought to evaluate the association between genetic variants in TCF7L2 with SU treatment failure in an independent cohort from Germany. We recruited 189 patients with T2D being treated with SU agents and determined the TCF7L2 rs7903146 diabetes
C Biomed Central

() 2011 Holstein et al; licensee BioMed Central Ltd. This is an Open Access article distributed under the terms of the Creative Commons Attribution License (http://creativecommons.org/licenses/by/2.0), which permits unrestricted use, distribution, and reproduction in any medium, provided the original work is properly cited. 
risk genotype, which has been reported as having the strongest association with T2D [3]. We used a logistic regression with secondary $\mathrm{SU}$ failure defined as an $\mathrm{A} 1 \mathrm{C}$ $\geq 7.0 \%$ after 6 months of SU treatment.

\section{Methods}

Subjects

One hundred and eighty-nine patients with T2D, all of them being treated with SU agents, were recruited at the medical department of the Klinikum Lippe-Detmold, a large tertiary care hospital in East Westphalia, Germany, between 1 January 2000 and 30.11.2009. As the only hospital in the area, the one at Lippe-Detmold is responsible for the inpatient and outpatient management of all emergencies in the region. All patients had been treated with the SU drugs glimepiride $(\mathrm{n}=147)$, glibenclamide $(\mathrm{n}=$ $39)$ and gliquidon $(n=3)$. Ninety-seven patients failed to respond to SU treatment according to our definition of A1C $\geq 7.0 \%$ after 6 months of treatment (76 patients treated with glimepiride, 19 with glibenclamide and 2 with gliquidon). Forty six patients were additionally treated with insulin. The mean $( \pm$ SD) daily dose of SU agents was comparable between subjects who failed to respond to SUs and the controls $(5.0 \pm 3.7 \mathrm{mg}$ vs. $6.8 \pm 3.7 \mathrm{mg}$, $P=0.13$ for glibenclamide; $2.5 \pm 1.6 \mathrm{mg}$ vs. $2.5 \pm 1.4 \mathrm{mg}$ for glimepiride, $P=0.99$ ). As our patients were recruited within the framework of a study originally investigating the risk of hypoglycaemia [17], eighty nine patients had experienced a severe hypoglycaemia, which was defined as a symptomatic event requiring treatment with intravenous glucose and was confirmed by a blood glucose measurement of $<50 \mathrm{mg} / \mathrm{dl}(<2.8 \mathrm{mmol} / \mathrm{l})$. Seventy-two subjects were additionally treated with insulin sensitizing drug metformin (32 patients in the control group and 40 patients in the group of patients with SU treatment failure; $P=0.36$, Table 1 ). The protocol was approved by the Ethics Committee of the University of Münster School of Medicine and by the Ethics Committee of the University of Leipzig, School of Medicine. All patients gave written informed consent to participate in the study.

\section{Genotyping of rs7903146}

Genotyping of rs7903146 in all study subjects was done using the TaqMan allelic discrimination assay (Assayson-Demand (TM), SNP Genotyping Products; Applied Biosystems, Inc.) on an ABI PRISM 7500 sequence detector (Applied Biosystems Inc.) according to the manufacturer's protocol. The genotype distribution was consistent with Hardy-Weinberg equilibrium $(P>0.05)$. Genotyping success rate was $>99 \%$, and duplicate genotyping concordance was $100 \%$.

\section{Statistics}

Standard descriptive and comparative statistics $\left(\chi^{2}\right.$ test, t-test) were used to characterize and compare clinical parameters in different groups (controls, cases). Logistic regression analyses were used to calculate the effects of investigated factors on SU treatment failure, which were reported as odds ratio with 95\% CI (confidence intervals). In the additive model, homozygotes for the major allele, heterozygotes and homozygotes for the minor allele were coded to a continuous numeric variable for genotype (as 0, 1, 2). Data were analyzed using the SPSS software package (version 15.0; SPSS, Inc., Chicago, IL).

\section{Results}

Clinical characteristics of all study participants are given in Table 1. As expected, the subjects with failure of SU treatment had a higher A1C than the controls (Table 1). However, both groups were comparable in regard to the age, gender, age at onset of diabetes, duration of diabetes and creatinine clearance and co-medication

Table 1 Clinical characteristics of all participants

\begin{tabular}{|c|c|c|c|}
\hline & Treatment with sulfonylurea (A1C $<7 \%$ ) & Failure of treatment with sulfonylurea $(\mathrm{A} 1 \mathrm{C} \geq 7 \%)$ & P-value \\
\hline & $N=92$ & $N=97$ & \\
\hline Gender (Male/Female) & $45 / 47$ & $47 / 50$ & $0.95^{*}$ \\
\hline Age $(y r)$ & $78.2 \pm 9.6$ & $78.3 \pm 9.0$ & 0.98 \\
\hline BMI $\left(\mathrm{kg} / \mathrm{m}^{2}\right)$ & $26.8 \pm 5.2$ & $27.0 \pm 4.7$ & 0.71 \\
\hline Creatinine (mg/dl) & $1.73 \pm 1.20$ & $1.52 \pm 0.55$ & 0.13 \\
\hline Creatinine clearence $(\mathrm{ml} / \mathrm{min})$ & $42.96 \pm 22.61$ & $42.10 \pm 18.77$ & 0.79 \\
\hline A1C (\%) & $6.13 \pm 0.51$ & $7.65 \pm 1.40$ & $<0.001$ \\
\hline Age at onset of diabetes (yr) & $68.0 \pm 13.4$ & $66.0 \pm 10.9$ & 0.28 \\
\hline Diabetes duration (yr) & $10.2 \pm 9.5$ & $11.9 \pm 8.4$ & 0.20 \\
\hline Co-medication ( $n$ all drugs) & $7 \pm 3$ & $7 \pm 3$ & 0.29 \\
\hline Sulfonylurea daily dose (mg) & $3.68 \pm 6.40$ & $4.24 \pm 6.85$ & 0.56 \\
\hline Metformin treatment ( $\mathrm{n}$ patients) & 32 & 40 & $0.36^{*}$ \\
\hline
\end{tabular}

Data are mean $\pm S D$; $P$ - values for comparisons between genotypic groups by ANOVA statistics.

* $\chi^{2}$ test. 
presented as number of drugs taken by the patient (Table 1). Also the number of patients additionally treated with metformin was similar between the groups (Table 1).

In the univariate logistic regression analyses, the TCF7L2 genotype was the only predictor of SU treatment failure (Table 2). The rs7903146 $\mathrm{T}$ allele was significantly more frequent in the group of patients who failed to respond to SU (36\%) than in the control group (26\%) $[P=0.046$; odds ratio (OR): $1.57(1.01-2.45)$ in an additive mode of inheritance] (Table 3 ). In the control group, $56.0 \%$ of subjects had the CC, $36.3 \%$ had the CT and $7.7 \%$ had the TT genotype. Among patients who failed to respond to SUs, $41.2 \%$ were CC homozygous, $46.4 \%$ were CT heterozygous and $12.4 \%$ were TT homozygous (Table 3).

To investigate whether the rs7903146 effect is specific to the mechanism of action of SUs, we evaluated the genotype effects on response to a non-insulin secretagogue metformin. By analysing 72 metformin-treated individuals only, no effect of the genotype on SU treatment failure was found $[P=0.98$; OR $1.01(0.50-2.03)]$.

\section{Secondary confirmatory analyses}

In secondary analyses we used a logistic regression with secondary SU failure defined as the addition of insulin after at least 6 months of SU therapy and corresponding A1C measurement of $\geq 7.0 \%$. Based on these criteria 46 patients from our cohort failed to respond to SU treatment and were additionally treated with insulin.

In the univariate logistic regression analyses including 46 patients who failed to respond to SU treatment and 143 control subjects, diabetes duration ( $<5$ yrs vs. $>5 \mathrm{yrs}$ ) appeared to be the strongest predictor of $\mathrm{SU}$ treatment failure [OR: 4.06 (1.50-11.01), $P=0.006$ ]. We also assessed the effect of rs7903146 on SU treatment failure. The rs7903146 T allele was significantly more frequent in the group of patients additionally treated with insulin $(40 \%)$ than in the control group treated only with SUs $(28 \%)[P=0.03$; odds ratio (OR): 1.73 (1.06-2.84) in an additive mode of inheritance]. In the

Table 2 Univariate regression analyses of predictors on failure of sulfonylurea treatment in patients with type 2 diabetes

\begin{tabular}{lcc}
\hline & Odds ratio (95\% Cl) & P-value \\
\hline Gender & $0.98(0.56-1.74)$ & 0.95 \\
Age $(y r)$ & $1.00(0.97-1.03)$ & 0.98 \\
$\begin{array}{l}\text { Diabetes duration (<5 yrs vs. } \\
>5 \text { yrs) }\end{array}$ & $1.65(0.86-3.17)$ & 0.13 \\
Sulfonylurea daily dose $(\mathrm{mg})$ & $1.01(0.97-1.06)$ & 0.57 \\
$\begin{array}{l}\text { TCF7L2 genotype (rs7903146; per } \\
\text { allele effect) }\end{array}$ & $1.57(1.01-2.45)$ & 0.046 \\
TCF7L2 genotype (CC vs. TT) & $2.09(1.02-4.27)$ & 0.043 \\
\hline
\end{tabular}

control group, $53 \%$ of subjects had the CC genotype, $39 \%$ had CT and $8 \%$ had TT. Among patients treated with insulin, $35 \%$ were CC homozygous, $50 \%$ were CT heterozygous and $15 \%$ had the TT genotype. The results remained materially unchanged even after including diabetes duration as a strong predictor of SU treatment failure in these analyses, thus indicating an independent effect of the genotype [OR: $1.66(0.99-2.79), P=0.06$ in additive model].

\section{Discussion}

In the present study, we investigated the effect of TCF7L2 diabetes risk T-allele at rs7903146 on therapeutic response to SUs. In univariate regression analyses, TCF7L2 genotype was the only predictor of SU treatment failure. The rs7903146 T-allele conferred a higher risk for sulfonylurea treatment failure as it was significantly more frequent in the group of patients who failed to respond to SUs (36\%) than in the control group (26\%). After adjusting for diabetes duration the odds ratio did not change $(\mathrm{OR}=1.57)$ and the $P$-value reduced just minimally (from $P=0.046$ to $P=0.057$ ), thus indicating independent effect of the TCF7L2 genotype. Despite the smaller sample size and so, limited statistical power, our data are in line with findings reported by Pearson et al. [15], suggesting that variation in TCF7L2 influences therapeutic response to SUs. Pearson et al. observed that homozygous carriers of the TCF7L2 risk alleles (rs1225372 and rs7903146) were twice as likely not to respond to SUs as patients homozygous for the non-risk alleles [15]. Considering pretreatment A1C levels as covariate in logistic regression analyses even strengthened the association between sulfonylurea response and genotype at rs7903146 [15]. Even though the findings are consistent between the present study and that reported by Pearson et al., several differences should be noted. First, Pearson et al. investigated a total of 911 SU users of 4,469 patients with T2D from the DARTS/MEMO (Diabetes Audit and Research Tayside/ Medicines Monitoring Unit) collaboration database, who were recruited to GoDARTS between 1997 and 2006 [15]. Second, SU failure was defined very restrictively as an $\mathrm{A} 1 \mathrm{C}>7 \%$ within 3-12 months after treatment initiation. According to these criteria, $42 \%$ of SU users failed to respond to the therapy [15]. In our study we chose a comparable, overlapping definition of secondary SU failure with at least 6 months of SU therapy and corresponding A1C measurement of $\geq 7.0 \%$. According to this definition, $51 \%$ of our patients failed to respond to SU therapy. We are aware that the difference in SU treatment failure frequency may be due to various study designs but it is noteworthy that to date, there is no widely accepted definition of secondary SU failure. Due to the lack of uniform definition, the frequency of SU 
Table 3 Effect of rs7903146 genotype on sulfonylurea treatment failure under logistic regression analysis

\begin{tabular}{|c|c|c|c|c|c|}
\hline Genotype rs7903146 & Total & $\begin{array}{l}\text { Controls } \\
\text { (A1C }<7 \%)\end{array}$ & $\begin{array}{l}\text { Failure of treatment with sulfonylurea } \\
\qquad(\mathrm{A} 1 \mathrm{C} \geq 7 \%)\end{array}$ & $\begin{array}{c}\text { Frequency of T-allele } \\
\text { (controls vs. treatment failure) }\end{array}$ & $\begin{array}{c}\text { Additive } \\
P \text { value } \\
\text { OR }(95 \% \mathrm{Cl})\end{array}$ \\
\hline & $n=188$ & $n=91$ & $n=97$ & & \\
\hline $\mathrm{CC}$ & $91(48.4 \%)$ & $51(56.0 \%)$ & $40(41.2 \%)$ & & \\
\hline CT & $78(41.5 \%)$ & 33 (36.3\%) & $45(46.4 \%)$ & $0.26 / 0.36$ & $1.57 \frac{\mathbf{0 . 0 4 6}}{(1.01-2.45)}$ \\
\hline$\pi$ & 19 (10.1\%) & $7(7.7 \%)$ & $12(12.4 \%)$ & & \\
\hline
\end{tabular}

failure varies considerably between $22 \%$ and $50 \%$ after 12 and 36 months of treatment, respectively $[18,19]$. The decreasing effectiveness of SUs results from progressive loss of $\beta$-cell function but also from patient related factors (dietary incompliance, weight gain, lack of exercise). Despite the above mentioned differences between the studies, frequencies of TT homozygous subjects in the groups of patients who failed to respond to the therapy (independent of definition) were significantly higher than in the control groups and were comparable between both studies $(12 \%$ vs. $8 \%$ in the German patients and $16 \%$ vs. $8 \%$ in the GoDARTS study). Also, genotype distribution of the rs7903146 was similar, with $10 \%$ and $11 \%$ of diabetic population with 2 copies of the T-allele in our study and the GoDARTS study, respectively. Finally, similarly to the GoDARTS study, our data suggest that carriers of the $\mathrm{T}$ allele were $57 \%$ more likely to fail SU treatment; TT homozygotes were twice as likely as $\mathrm{CC}$ homozygotes.

It is noteworthy that an alternate definition of SU treatment failure in our cohort based on addition of insulin after at least 6 months of SU therapy and corresponding $\mathrm{A} 1 \mathrm{C}$ measurement of $\geq 7.0 \%$ yielded similar results. Even though not independent from the previous analyses, these findings provide further support for the role of TCF7L2 genotypes in altered hypoglycaemic response to SUs. Interestingly, when using this definition of SU treatment failure, diabetes duration appeared to be a predictor of treatment failure along with the TCF7L2 genotype. Nevertheless, the genotype effect was independent as even after adjustment for diabetes duration, the results remained materially unchanged. Although the $P$-value went from 0.04 to 0.06 the odds ratio reduced only minimally (from 1.73 to 1.66 ).

Indirectly, our findings also support studies that favour the role of TCF7L2 in the regulation of insulin secretion. However, we are aware that since the TCF7L2 variants increase progression from IGT to diabetes [5], additional models considering diabetes therapy, particularly including a control group having been treated with a different antidiabetic agent - e.g., metformin, would be desirable to clarify whether the observed data reflect pharmacogenetic effects specific to SUs or rather a disease-genetic process. Indeed, such a control group treated with metformin was included in the GoDARTS study [15]. The study suggested pharmacogenetic effects of TCF7L2 SNPs influencing therapeutic response to sulfonylureas but not metformin, since no association was seen between metformin response and TCF7L2 variants [15]. Even though limited by the small sample size $(\mathrm{N}=72)$, we also failed to observe any influence of TCF7L2 genotypes on the response to metformin, as a non-insulin secretagogue, thus further supporting the notion that TCF7L2 effect is specific to the mechanism of action of SUs.

One of the major limitations of our study is the low sample size and so, limited statistical power. Taking into account genotype frequencies and the sample size in our study we had a statistical power of $80 \%$ (at $\alpha=$ 0.05 ) to detect genetic risk (odds ratio) of 1.8 for treatment failure in additive mode of inheritance (software Quanto version 1.2.2) [20]. In contrast, the GoDARTS study by Pearson et al. [15] had $80 \%$ power to detect risk (OR) as low as 1.3. Also noteworthy, given the TCF7L2 diabetes risk genotypes make patients more resistant to the action of sulfonylureas, one would expect that carriers of the risk genotype should be less likely to become hypoglycaemic. However, in our study we could not observe any differences in the frequency of the diabetes risk allele between patients with and without hypoglycaemia $(P=0.30)$.

\section{Conclusion}

In conclusion, present data strengthen previously reported findings suggesting altered therapeutic response to SUs in patients with T2D carrying the diabetes risk alleles at TCF7L2 variants.

\section{Acknowledgements}

This work was supported by grants from the Deutsche

Forschungsgemeinschaft (KFO-152 "Atherobesity" to AK (KO3512/1-1) and DHFD to MS, PK). We would like to thank Ines Müller and Beate Enigk for their excellent technical assistance.

\section{Author details}

${ }^{1}$ First Department of Medicine, Clinic Lippe-Detmold, Detmold, Germany. ${ }^{2}$ University Hospital for Children and Adolescents, University of Leipzig, 
Leipzig, Germany. ${ }^{3}$ Department of Medicine, University of Leipzig, Leipzig, Germany. ${ }^{4}$ Interdisciplinary Centre for Clinical Research, University of Leipzig, Leipzig, Germany.

\section{Authors' contributions}

$\mathrm{MH}$ was responsible for clinical characteristics of study subjects. AK and MS edited the manuscript and contributed to the discussion. AH and PK conceived and designed the study and wrote the manuscript. All authors read and approved the final manuscript.

\section{Competing interests}

The authors declare that they have no competing interests.

Received: 8 October 2010 Accepted: 24 February 2011

Published: 24 February 2011

\section{References}

1. Clatworthy JP, Subramanian V: Stem cells and the regulation of proliferation, differentiation and patterning in the intestinal epithelium: emerging insights from gene expression patterns, transgenic and gene ablation studies. Mechanisms of Development 2001, 101:3-9.

2. Grant SF, Thorleifsson G, Reynisdottir I, Benediktsson R, Manolescu A, Sainz J, Helgason A, Stefansson H, Emilsson V, Helgadottir A, Styrkarsdottir U, Magnusson KP, Walters GB, Palsdottir E, Jonsdottir T, Gudmundsdottir T, Gylfason A, Saemundsdottir J, Wilensky RL, Reilly MP, Rader DJ, Bagger Y, Christiansen C, Gudnason V, Sigurdsson G, Thorsteinsdottir U, Gulcher JR, Kong A, Stefansson K: Variant of transcription factor 7-like 2 (TCF7L2) gene confers risk of type 2 diabetes. Nat Genet 2006, 38:320-323.

3. Zeggini E, McCarthy Ml: TCF7L2: the biggest story in diabetes genetics since HLA? Diabetologia 2007, 50:1-4.

4. Cauchi S, El Achhab YE, Choquet H, Dina C, Krempler F, Weitgasser R, Nejjari C, Patsch W, Chikri M, Meyre D, Froguel P: TCF7L2 is reproducibly associated with type 2 diabetes in various ethnic groups: a global metaanalysis. J Mol Med 2007, 85:777-782.

5. Florez JC, Jablonski KA, Bayley N, Pollin TI, de Bakker PI, Shuldiner AR, Knowler WC, Nathan DM, Altshuler D: TCF7L2 polymorphisms and progression to diabetes in the Diabetes Prevention Program. N Engl J Med 2006, 355:241-250.

6. Kimber CH, Doney AS, Pearson ER, McCarthy MI, Hattersley AT, Leese GP, Morris AD, Palmer CN: TCF7L2 in the Go-DARTS study: evidence for a gene dose effect on both diabetes susceptibility and control of glucose levels. Diabetologia 2007, 50:1186-1191.

7. Körner A, Berndt J, Stumvoll M, Kiess W, Kovacs P: TCF7L2 gene polymorphisms confer an increased risk for early impairment of glucose metabolism and increased height in obese children. J Clin Endocrinol Metab 2007, 92:1956-1960.

8. Damcott CM, Pollin TI, Reinhart LJ, Ott SH, Shen H, Silver KD, Mitchell BD, Shuldiner AR: Polymorphisms in the transcription factor 7-like 2 (TCF7L2) gene are associated with type 2 diabetes in the Amish - Replication and evidence for a role in both insulin secretion and insulin resistance. Diabetes 2006, 55:2654-2659.

9. Saxena R, Gianniny L, Burtt NP, Lyssenko V, Giuducci C, Sjogren M, Florez JC, Almgren P, Isomaa B, Orho-Melander M, Lindblad U, Daly MJ, Tuomi T, Hirschhorn JN, Ardlie KG, Groop LC, Altshuler D: Common single nucleotide polymorphisms in TCF7L2 are reproducibly associated with type 2 diabetes and reduce the insulin response to glucose in nondiabetic individuals. Diabetes 2006, 55:2890-2895.

10. Scott LJ, Bonnycastle LL, Willer CJ, Sprau AG, Jackson AU, Narisu N, Duren WL, Chines PS, Stringham HM, Erdos MR, Valle TT, Tuomilehto J, Bergman RN, Mohlke KL, Collins FS, Boehnke M: Association of transcription factor 7-like 2 (TCF7L2) variants with type 2 diabetes in a Finnish sample. Diabetes 2006, 55:2649-2653.

11. Munoz J, Lok KH, Gower BA, Fernandez JR, Hunter GR, Lara-Castro C, De Luca M, Garvey WT: Polymorphism in the transcription factor 7-like 2 (TCF7L2) gene is associated with reduced insulin secretion in nondiabetic women. Diabetes 2006, 5:3630-3634.

12. Cauchi S, Meyre D, Dina C, Choquet H, Samson C, Gallina S, Balkau B, Charpentier G, Pattou F, Stetsyuk V, Scharfmann R, Staels B, Fruhbeck G, Froguel P: Transcription factor TCF7L2 genetic study in the French population - Expression in human beta-cells and adipose tissue and strong association with type 2 diabetes. Diabetes 2006, 55:2903-2908.

13. Wang J, Kuusisto J, Vänttinen $M$, Kuulasmaa T, Lindström J, Tuomilehto J, Uusitupa M, Laakso M: Variants of transcription factor 7-like 2 (TCF7L2) gene predict conversion to type 2 diabetes in the Finnish Diabetes Prevention Study and are associated with impaired glucose regulation and impaired insulin secretion. Diabetologia 2007, 50:1192-1200.

14. Loos RJF, Franks PW, Francis RW, Barroso I, Gribble FM, Savage DB, Ong KK, O'Rahilly S, Wareham NJ: TCF7L2 polymorphisms modulate proinsulin levels and beta-cell function in a British europid population. Diabetes 2007, 56:1943-1947.

15. Pearson ER, Donnelly LA, Kimber C, Whitley A, Doney AS, McCarthy MI, Hattersley AT, Morris AD, Palmer CN: Variation in TCF7L2 influences therapeutic response to sulfonylureas: A GoDARTs study. Diabetes 2007, 56:2178-2182.

16. Gong MN: Gene association studies in acute lung injury: replication and future direction. Am J Physiol 2009, 296:L711-L712.

17. Holstein A, Hahn M, Stumvoll M, Kovacs P: The E23K variant of KCNJ11 and the risk for severe sulfonylurea-induced hypoglycemia in patients with type 2 diabetes. Horm Metab Res 2009, 41:387-390.

18. Boccuzzi SJ, Wogen J, Fox J, Sung JC, Shah AB, Kim J: Utilization of oral hypoglycemic agents in a drug-insured US population. Diab Care 2001, 24:1411-1415.

19. Turner RC, Cull CA, Frighi V, Holman RR: Glycemic control with diet, sulfonylurea, metformin, or insulin in patients with type 2 diabetes mellitus: progressive requirement for multiple therapies (UKPDS 49). UK Prospective Diabetes Study (UKPDS) Group. JAMA 1999, 281:2005-2012.

20. Gauderman WJ, Morrison JM: QUANTO 1.1: A computer program for power and sample size calculations for genetic-epidemiology studies. 2006 [http://hydra.usc.edu/gxe]

\section{Pre-publication history}

The pre-publication history for this paper can be accessed here: http://www.biomedcentral.com/1471-2350/12/30/prepub

\section{doi:10.1186/1471-2350-12-30}

Cite this article as: Holstein et al:: TCF7L2 and therapeutic response to sulfonylureas in patients with type 2 diabetes. BMC Medical Genetics 2011 12:30.

\section{Submit your next manuscript to BioMed Central and take full advantage of:}

- Convenient online submission

- Thorough peer review

- No space constraints or color figure charges

- Immediate publication on acceptance

- Inclusion in PubMed, CAS, Scopus and Google Scholar

- Research which is freely available for redistribution 